\title{
Note on Transliteration and Translation
}

This book follows the transliteration system of the International Journal of Middle East Studies except that diacritical marks have been omitted. All translations are mine unless otherwise noted. 
This page intentionally left blank 